Revue Gouvernance

Governance Review

GOUVERNANCE

GOU ERNAN CLE

\title{
Vers l'émergence d'une gouvernance territoriale régionale autochtone ? Parcours des Mi'gmaq de Gespeg pour transformer la gestion des forêts publiques de leur territoire ancestral au Québec, Canada
}

\author{
Denis Blouin, Jean-François Bissonnette et Luc Bouthillier
}

Volume 17, numéro 2, 2020

Communautés dépendantes des forêts et gouvernance

Forest Dependent Communities and Governance

URI : https://id.erudit.org/iderudit/1073112ar

DOI : https://doi.org/10.7202/1073112ar

Aller au sommaire du numéro

Éditeur(s)

Centre d'études en gouvernance de l’Université d'Ottawa

ISSN

1912-0362 (numérique)

Découvrir la revue

Citer cet article

Blouin, D., Bissonnette, J.-F. \& Bouthillier, L. (2020). Vers l'émergence d'une gouvernance territoriale régionale autochtone ? Parcours des Mi'gmaq de Gespeg pour transformer la gestion des forêts publiques de leur territoire ancestral au Québec, Canada. Revue Gouvernance / Governance Review, 17(2), 78-104. https://doi.org/10.7202/1073112ar
Résumé de l'article

Les Mi'gmaq de Gespeg sont une communauté autochtone sans territoire attribué. Cette situation amplifie chez elle une volonté de réappropriation du territoire et de ses ressources présente chez les Premières Nations du Canada. Cette volonté s'est entre autres incarnée dans des ententes de cogestion forestière en terre publique avec le gouvernement du Québec. Pour comprendre ce processus transformatif, nous avons réalisé une étude de cas fondée sur une approche collaborative et partenariale avec les Mi'gmaq de Gespeg. Après avoir défini leurs attentes et leur vision face à la forêt, nous avons retracé leur contexte sociohistorique forestier, puis analysé leur cheminement en foresterie au cours des vingt dernières années, et plus particulièrement leur engagement dans la cogestion. Nous avons ensuite développé un cadre d'analyse de la gouvernance forestière, en identifiant plus particulièrement les conditions sur lesquelles la communauté peut agir pour concrétiser sa vision. Nous avons constaté que l'engagement de la communauté en foresterie semble un moyen mis de l'avant pour entamer la transformation de la gouvernance de son territoire forestier ancestral. Ce cheminement stratégique circonscrit par le cadre de la cogestion constituerait un passage vers une gouvernance territoriale autochtone à Gespeg, en cogestion avec des partenaires locaux, la communauté mig’maq y assumant un rôle d'entrepreneur institutionnel. Les Mi'gmaq de Gespeg exerceraient ainsi une fonction d'agent transformatif sur les conditions actionnables de la gouvernance des terres publiques vers l'atteinte de leur vision de gouvernance du territoire ancestral.
(C) Denis Blouin, Jean-François Bissonnette et Luc Bouthillier, 2020
Ce document est protégé par la loi sur le droit d'auteur. L'utilisation des services d'Érudit (y compris la reproduction) est assujettie à sa politique d'utilisation que vous pouvez consulter en ligne. 


\section{Vers l'émergence d'une gouvernance territoriale régionale autochtone? Parcours des Mi'gmaq de Gespeg pour transformer la gestion des forêts publiques de leur territoire ancestral au Québec, Canada.}

Par Denis Blouin ${ }^{1}$, Jean-François Bissonnette ${ }^{2}$ et Luc Bouthillier ${ }^{3}$

\section{Résumé}

Les Mi'gmaq de Gespeg sont une communauté autochtone sans territoire attribué. Cette situation amplifie chez elle une volonté de réappropriation du territoire et de ses ressources présente chez les Premières Nations du Canada. Cette volonté s'est entre autres incarnée dans des ententes de cogestion forestière en terre publique avec le gouvernement du Québec. Pour comprendre ce processus transformatif, nous avons réalisé une étude de cas fondée sur une approche collaborative et partenariale avec les Mi'gmaq de Gespeg. Après avoir défini leurs attentes et leur vision face à la forêt, nous avons retracé leur contexte sociohistorique forestier, puis analysé leur cheminement en foresterie au cours des vingt dernières années, et plus particulièrement leur engagement dans la cogestion. Nous avons ensuite développé un cadre d'analyse de la gouvernance forestière, en identifiant plus particulièrement les conditions sur lesquelles la communauté peut agir pour concrétiser sa vision. Nous avons constaté que l'engagement de la communauté en foresterie semble un moyen mis de l'avant pour entamer la transformation de la gouvernance de son territoire forestier ancestral. Ce cheminement stratégique circonscrit par le cadre de la cogestion constituerait un passage vers une gouvernance territoriale autochtone à Gespeg, en cogestion avec des partenaires locaux, la communauté mig'maq y assumant un rôle d'entrepreneur institutionnel. Les Mi'gmaq de Gespeg exerceraient ainsi une fonction d'agent transformatif sur les conditions actionnables de la gouvernance des terres publiques vers l'atteinte de leur vision de gouvernance du territoire ancestral.

Mots-clés : Mi'gmaq, foresterie autochtone, cogestion forestière, gouvernance territoriale et forestière, agentivité, entrepreneur institutionnel

1. Denis Blouin est doctorant en sciences forestières au Département des sciences du bois et de la forêt de l'Université Laval. Il est membre de l'Ordre des urbanistes du Québec.

2. Jean-François Bissonnette est professeur adjoint au Département de géographie de l'Université Laval et chercheur membre du Centre des sciences sur la biodiversité du Québec (CSBQ).

3. Luc Bouthillier est professeur titulaire au Département des sciences du bois et de la forêt de l'Université Laval. 


\section{Abstract}

The Mi'gmaq of Gespeg are an aboriginal community with no assigned territory. This situation amplifies their desire to reclaim the territory and its resources encountered by First Nations in Canada. This desire has been embodied in forest co-management agreements with the Government of Quebec. To understand this transformative process, we carried out a case study based on a collaborative and partnership approach with the Mi'gmaq of Gespeg. Following the identification of their expectations and their vision about forest, we considered their socio-historical forest context, then analyzed their progress in forestry over the last twenty years, more particularly their commitment to co-management. We then developed a framework for analyzing forest governance, identifying in particular the conditions on which the community can act to realize its vision. We have found that community involvement in forestry seems to be a means to begin transforming the governance of their ancestral forest land. This strategic pathway, circumscribed by the co-management framework, would constitute a transition to indigenous territorial governance in Gespeg, in co-management with local partners, in which the mig'maq community assume an institutional entrepreneur role. The Mi'gmaq of Gespeg would thus exercise a function of transformative agent on the actionable conditions of the governance of the public lands towards the achievement of their vision of governance of the ancestral territory.

Keywords : Mi'gmaq, aboriginal forestry, forest co-management, land and forest governance, agency, institutional entrepreneur

\section{Introduction}

Le territoire forestier est un enjeu majeur pour plusieurs communautés canadiennes, et particulièrement pour les Premières Nations, dont il constitue le milieu de vie et un fondement identitaire (Beaudoin et coll., 2016). Au cours des derniers siècles suivant la colonisation européenne, les forêts canadiennes ont connu de profonds changements écologiques et sociopolitiques. Ces transformations ont eu des impacts considérables sur le mode de subsistance des communautés autochtones et sur leur relation au territoire forestier. Suivant un parcours et un horizon de temps différents pour chaque Première Nation, un mode de vie centré sur le nomadisme et l'autosubsistance est devenu impraticable à l'échelle de communautés entières. Les phénomènes qui se sont succédé, tels les politiques coloniales, la sédentarisation, le mode de gouvernance étatique des ressources naturelles et du territoire, et les transformations technico-économiques, ont redéfini le cadre d'existence des communautés autochtones forestières.

Pendant les dernières décennies, l'implication des communautés autochtones dans la gouvernance forestière a connu une évolution manifeste (Beaudoin et coll., 2012; Wyatt et coll., 2010). D'une dépossession plus ou moins complète des Premières Nations, la situation actuelle favorise désormais la mise en place de différentes modalités de 
cogestion forestière (Hébert, 2006). Ces modalités et leur processus de mise en ouvre ne sont que partiellement compris compte tenu de la diversité des situations au Canada (Fortier et Wyatt, 2019).

La spécificité des contextes soulève un questionnement sur les choix stratégiques faits par les communautés quant à leur acceptation ou leur contestation des modèles gouvernementaux de cogestion forestière, notamment en ce qui concerne la construction de leurs objectifs (Wyatt et coll., 2019). Prenant la balle au bond, cet article a pour but d'explorer le cheminement de la Nation micmac de Gespeg ${ }^{4}$ en foresterie afin de comprendre comment il contribue à affirmer l'identité de la communauté sur son territoire traditionnel et permet d'envisager un nouveau mode de gouvernance.

Actuellement, les limites du cadre de la gestion des forêts au Québec ne correspondent pas aux attentes et à la vision des Mi'gmaq de Gespeg (Blouin et coll., 2020). Toutefois, les autorités communautaires ont jugé bon d'inscrire certaines actions dans ce cadre, malgré les contraintes rencontrées. Pourquoi ont-ils pris cette décision et à quelle fin? Est-ce une stratégie consciente et proactive afin de progresser vers un objectif précis, ou simplement une approche opportuniste, une occasion offerte qui aurait éveillé un intérêt ponctuel pour la foresterie?

Sur la base de ces questions, nos objectifs sont les suivants:

1) caractériser le cheminement forestier des Mi'gmaq de Gespeg, plus particulièrement dans la gouvernance forestière actuelle;

2) identifier les conditions modelant l'engagement mi'gmaq en foresterie.

Ces objectifs nous permettront de mettre en exergue les tenants et aboutissants de l'engagement en foresterie de la communauté de Gespeg en relation avec ses transformations sociopolitiques. Premièrement, nous présenterons le cadre conceptuel élaboré pour les fins de cette recherche. Ensuite, nous décrirons l'évolution historique de la communauté de Gespeg. Puis, nous insisterons sur ses engagements en foresterie et son rôle dans la gouvernance forestière. Cela nous permettra d'aborder les notions de cogestion et de gouvernance, ainsi que d'envisager des liens conceptuels avec les notions d'agentivité et d'entrepreneur institutionnel.

4. Nation micmac de Gespeg est la dénomination officielle du conseil de bande auprès des instances gouvernementales. La communauté de Gespeg emploie le gentilé Mi'gmaq pour se définir. 


\section{Cadre conceptuel}

Depuis des millénaires, le territoire mi'gmaq constitue un système socioécologique complexe transformé par les événements. Nous nous intéressons à l'évolution du milieu de vie forestier des Mi'gmaq de Gespeg. La portion dans laquelle se manifeste leur engagement en foresterie, les vingt dernières années, fera l'objet d'une analyse détaillée.

La définition de la gouvernance employée dans cette étude se veut large et englobante. Nous nous référerons plus particulièrement à celle de Arts et Visseren-Hamakers (2012, p. 4), pour qui la gouvernance désigne les «différentes façons, qu'elles soient autonomes ou réciproques, dont les acteurs publics et privés, autant ceux liés à l'État qu'au marché et à la société civile, gèrent les questions publiques». Cette définition de la gouvernance permet d'englober de multiples formes d'arrangements institutionnels (Arts, 2014), comme les différentes formes de cogestion existantes en foresterie au Québec. Nous considérerons la gouvernance globale de la forêt gaspésienne, en nous attardant surtout à l'engagement des Mi'gmaq de Gespeg dans celle-ci sous la forme d'ententes de cogestion.

La cogestion est un système de gouvernance considérée, entre autres, comme favorisant l'autonomie des Premières Nations dans la gestion des ressources et de leur territoire (Fuss et coll., 2018). Elle se caractérise par "le partage des pouvoirs et des responsabilités entre le gouvernement et les utilisateurs locaux de la ressource „ ${ }^{6}$ (Berkes, 2009, p. 1692). Nous examinerons ce partage entre le gouvernement du Québec et la communauté de Gespeg, ainsi que la satisfaction de celle-ci face à cette entente.

Notre cadre d'analyse du système de gouvernance forestière à Gespeg s'inspire, entre autres, de celui développé par Elinor Ostrom, soit le institutional analysis and development (IAD) framework (Ostrom, 1990), et des propositions de modifications faites par Floriane Clement pour lui adjoindre une dimension politisée (Clement, 2010). Son utilisation est jugée hautement efficace pour analyser la cogestion des ressources naturelles (Whaley et Weatherhead, 2014). Le social-ecological systems framework (SES) d'Ostrom (2007) a aussi servi de référence, par l'entremise de la proposition de cadre combinée élaborée par Daniel H. Cole et ses collaborateurs (2019) aux fins de corriger leurs lacunes respectives. Ces cadres d'analyse sont largement employés dans l'analyse de la gouvernance des ressources naturelles et de leurs processus de transformation (Cole et coll., 2019; Binder et coll., 2013).

5. Traduction libre des auteurs

6. Traduction libre des auteurs 
Concernant l'étude des communautés canadiennes dépendantes des ressources naturelles, telle Gespeg, une revue de littérature récente (Teitelbaum et coll., 2019) met en lumière l'importance d'intégrer des concepts théoriques liés au contexte global dans lequel s'insère le territoire à l'étude pour mieux saisir les processus et les dynamiques à l'œuvre. Nous croyons que l'ajout dans le cadre d'analyse du contexte politicoéconomique et du discours, envisagé en tant que modeleur des croyances, des valeurs et des normes (Clement, 2010), est une réponse valide à cette recommandation.

Cependant, nous apportons d'autres modifications à ce cadre d'analyse en y intégrant des notions issues de la "theory of access" (Ribot et Peluso, 2003). Cette approche analytique est utile à la compréhension des mécanismes à l'œuvre dans la répartition entre les différents acteurs de l'accès au territoire et aux bénéfices de ses ressources (Myers et Hansen, 2019). Ainsi, nous caractériserons la trajectoire historique, les stratégies et les outils qu'utilisent les Mi'gmaq pour accéder au territoire et à ses ressources, ainsi que pour confronter le contexte existant (Peluso et Lund, 2011). Plus particulièrement, notre cadre d'analyse vise à faciliter la compréhension du vécu des Mi'gmaq de Gespeg à travers le temps et de leur posture concernant leur territoire ancestral et sa gouvernance forestière. La figure 1 représente une schématisation de notre cadre d'analyse.

\section{Figure 1 : Schéma du cadre d'analyse de la gouvernance du territoire et de ses ressources}

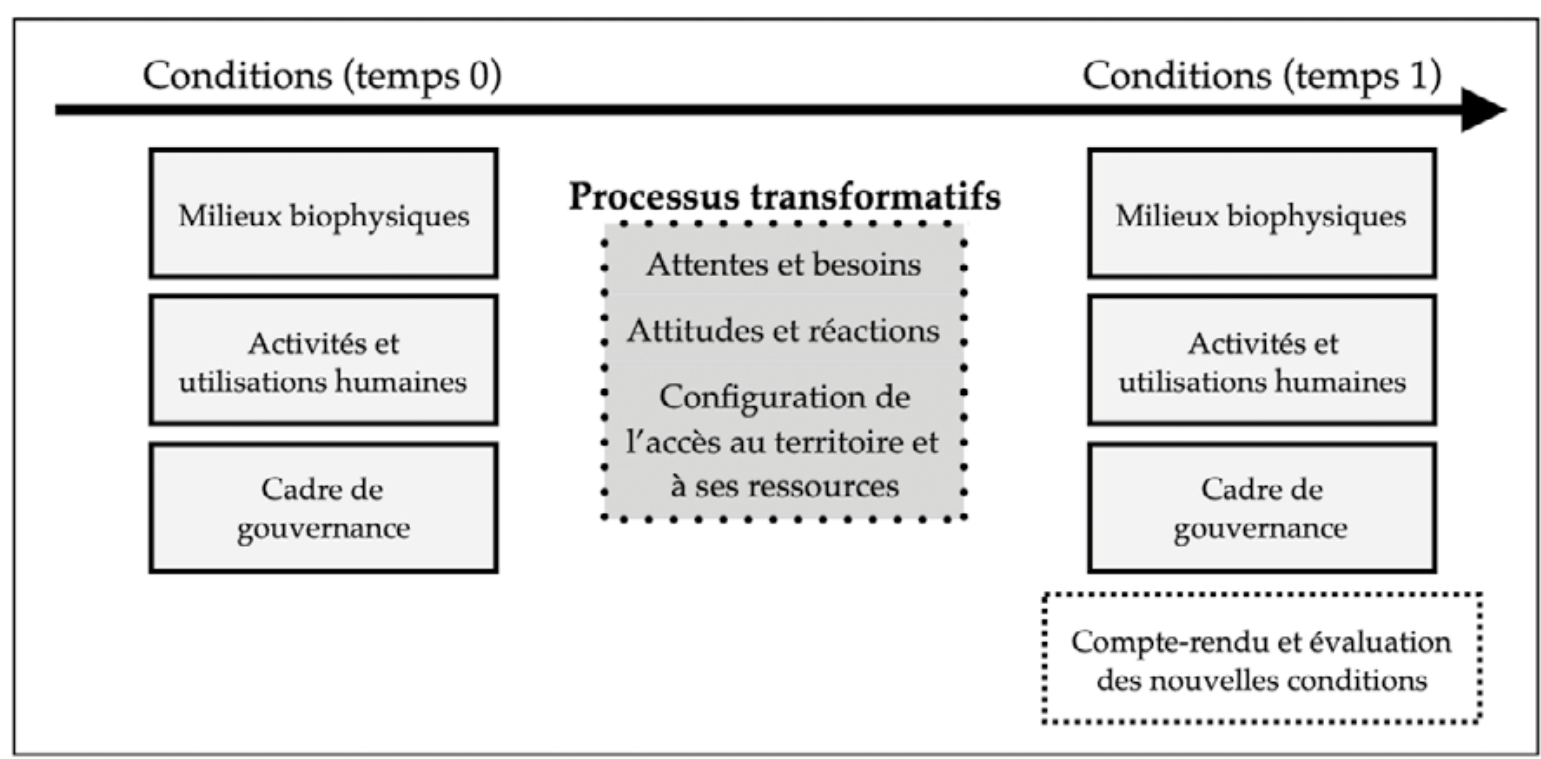

Inspiré de Cole et coll. (2019)

À partir de ce cadre schématique (figure 1) et conceptuel, nous avons construit un cadre d'analyse détaillé (figure 2), que nous avons ajusté de manière inductive en cours de recherche. Nous l'emploierons pour structurer notre analyse. 
Figure 2 : Cadre d'analyse de la gouvernance du territoire et de ses ressources

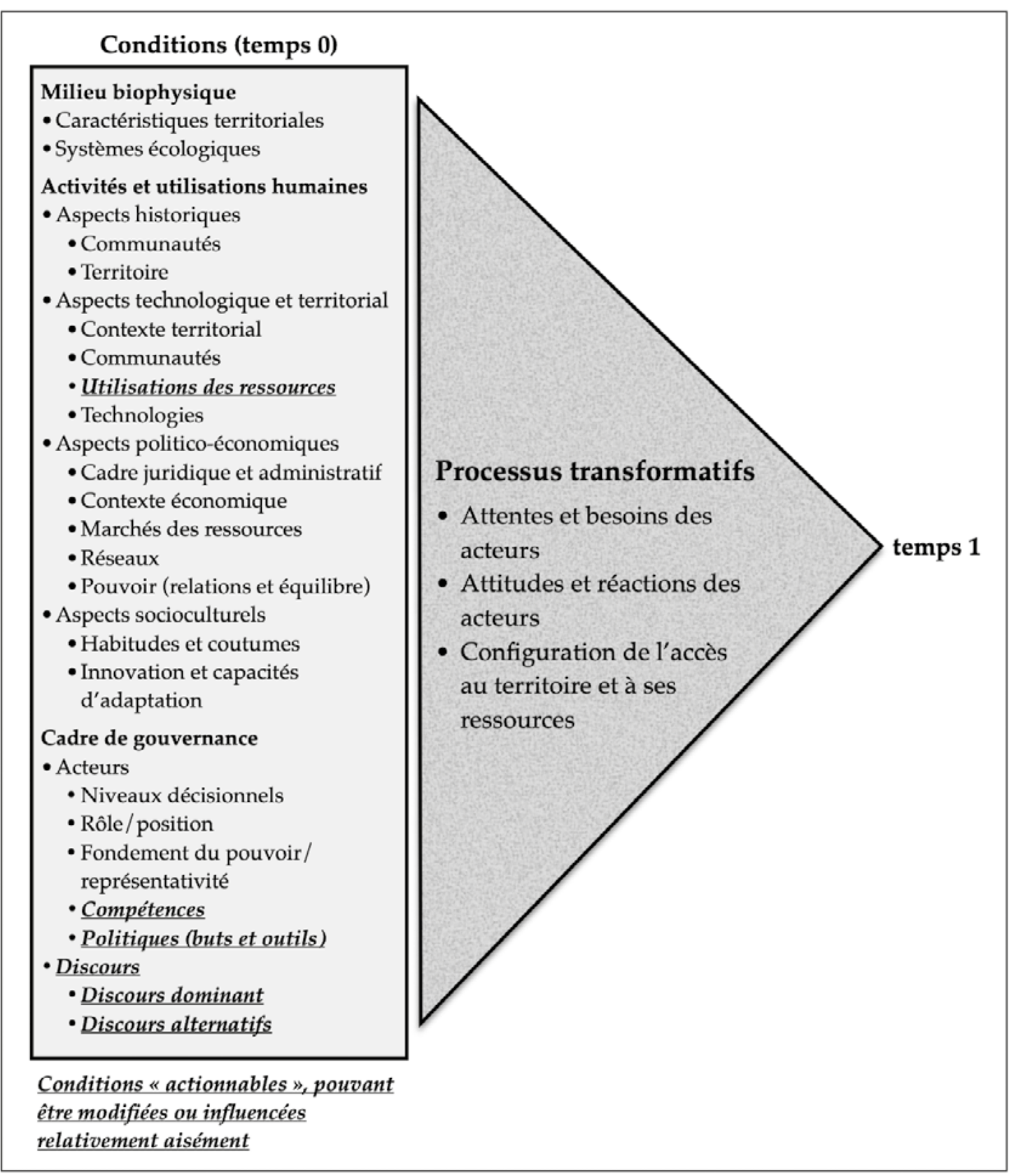

Un point distinctif de celui-ci est que nous y avons introduit la notion de conditions actionnables. En effet, les conditions existantes à un temps donné d'un système de gouvernance sont, dans une forte proportion, quasi immuables ou très peu transformables (Brusati et coll., 2016). Nous identifierons donc les conditions selon qu'elles ne peuvent pas être modifiées ou influencées, sinon très difficilement, ou qu'elles peuvent être modifiées ou influencées relativement aisément. Ainsi, l'agent transformatif ne peut exercer son agentivité qu'en influant sur les conditions actionnables de la gouvernance. 
Pour notreétude, nous considérerons la Nation micmac de Gespeg en tant qu'agent transformatif revêtant l'habit d'un entrepreneur institutionnel (Levy et Scully, 2007). Suivant cette logique, l'entrepreneur institutionnel qui souhaite induire des transformations réussies sur la gouvernance d'un système socioécologique doit appuyer sa stratégie sur des actions agissant sur les conditions actionnables (Westley et coll., 2013).

\section{Méthodologie}

Notre recherche est fondée sur l'emploi d'une approche partenariale et collaborative (Bourassa et coll., 2007; Tremblay et Demers, 2018). Cette méthodologie partenariale s'inscrit dans une étude de cas (Yin, 2014). Cette approche correspond aux attentes et aux intérêts des Premières Nations concernant les projets de recherche qui les touchent (Asselin et Basile, 2018), car elle permet de générer des connaissances et des retombées profitables à la communauté participante. À travers les échanges entre les acteurs et les chercheurs, elle permet une co-construction de connaissances sur : 1) l'étude des enjeux de la communauté et l'analyse de la situation; 2) la définition du problème; et 3) la conception d'un scénario de solution au problème vécu (Foudriat et coll., 2000).

La communauté mi'gmaq de Gespeg et son territoire ancestral constituent l'unité d'analyse. Cette communauté se distingue par certaines caractéristiques. Il s'agit de la seule communauté autochtone au Québec ayant un statut officiel, mais sans territoire attribué en réserve. De même, la majorité des membres de la Nation mi'gmaq de Gespeg vivent à l'extérieur du territoire ancestral, dont plus de la moitié dans la région montréalaise où ils disposent d'un centre communautaire.

Les méthodes utilisées pour mener cette recherche selon une approche collaborative et partenariale sont la réalisation d'entrevues non structurées (plus d'une centaine) avec des personnes-clés (élus, employés du conseil, représentants du gouvernement ou d'organismes), l'étude de la littérature grise (documents écrits de la communauté, documents gouvernementaux, documents d'organismes, documents juridiques), ainsi qu'une recherche-action participative. Par l'utilisation de ces méthodes, nous cherchions à connaître l'historique forestier de la communauté, à comprendre son implication en foresterie, puis à définir son projet forestier. Nous avons analysé le contenu des documents et des entretiens en parallèle à la participation au processus de co-construction avec la communauté de Gespeg.

Dans la recherche-action, nous avons analysé les actions suivant un processus continu inspiré du modèle "participation-définition-intervention" (Moisdon, 1984). Un processus itératif de ce genre permet d'accéder aux données de la recherche en participant au 
processus de définition du problème avec la communauté. De même, les options de solutions coconstruites y subissent une validation au contact des autres parties prenantes concernées (organismes locaux et gouvernements) par des échanges ultérieurs.

Dans le cadre de la démarche de recherche, nous avons effectué 11 séjours dans la communauté de Gespeg pour y tenir des rencontres de travail et d'échanges. Plusieurs dizaines d'appels et de conférences téléphoniques ainsi que plus d'une centaine de courriels ont été nécessaires pour réaliser ce projet de recherche en collaboration avec la Gmu'ge'qagua, soit la division foresterie du Conseil de bande de Gespeg. Tous ces contacts directs avec les Mi'gmaq de Gespeg et les différents acteurs impliqués ont permis de recueillir des observations directes de février 2016 à juin 2019. Un journal de bord a été tenu pour y consigner les observations effectuées par le chercheur dans le cadre de ces visites (Roy, 2016). Cet outil nous a permis de mettre en relation les éléments recueillis tout au long du projet de recherche. L'ensemble de la démarche impliquait aussi des rencontres avec le conseil de bande pour obtenir des rétroactions et une validation. Ce processus de co-construction est encore en cours au moment de rédiger cet article.

\section{Contexte sociohistorique d'une Première Nation mi'gmaq}

\subsection{Des semi-nomades : autosubsistance et trappe}

Occupant la péninsule gaspésienne depuis quelques milliers d'années, la société mi'gmaq repose sur un système de subsistance fondé sur la pêche, la chasse et la cueillette, conjugué à un mode de vie semi-nomade (Mawiomi de Gespe'gawa'gi, 2018). Les rivières à saumon et leur embouchure constituent le cour de son milieu de vie. La forêt est surtout fréquentée en hiver pour la chasse au gros gibier, ainsi que pour la récolte de matériaux et de plantes.

L'arrivée des pêcheurs européens et des explorateurs français amorce une période d'échanges commerciaux associés à la fourrure et à la pêche. La trappe prend alors une importance accrue dans le mode de vie mi'gmaq et les fourrures sont échangées aux commerçants européens contre divers objets utilitaires et ornementaux. L'occupation européenne plutôt éparse se limite alors à des postes de traite et à des installations de pêche généralement saisonnières. 


\subsection{Des vagabonds, la survivance comme mode de vie}

La fin du 18e siècle marque l'amplification du peuplement européen en Gaspésie avec l'arrivée des Acadiens, puis des Loyalistes et des commerçants anglo-normands. La situation des Mi'gmaq se marginalise avec l'empiètement sur leur milieu de vie et leur territoire. "Dès le début de la soi-disant souveraineté britannique (1763), une forme européenne de développement économique a pris forme, et une occupation territoriale bien plus envahissante a commencé. Ajoutons à ces deux changements la dépossession de la population mi'gmaq qui devient de plus en plus tragique pour nous au cours de ces siècles.» (Mawiomi de Gespe'gawa'gi, 2018, p. 162). Le territoire se transforme sous l'effet de l'agriculture, de l'exploitation forestière et des pêcheries commerciales. Cela induit une dépossession amplifiée par l'absence de droits reconnus par la Couronne britannique (Desjardins, 1981, dans Mawiomi de Gespe'gawa'gi, 2018; Fuss et coll., 2018).

C'est au début du $19^{\text {e }}$ siècle que démarre l'exploitation commerciale de la forêt, dans le but d'approvisionner les chantiers de construction navale et le commerce du bois équarri, surtout en pin blanc (Pinna et coll., 2009). Vers 1850, l'exploitation forestière en Gaspésie commence à s'orienter vers la production de bois de sciage, ce qui entraîne une diversification des essences recherchées (MFFP, 2017a). Le flottage du bois sur les rivières débute à cette époque.

En 1851, lors du processus d'attribution des territoires de réserves au Bas-Canada, les autorités coloniales qualifient les Mig'maq de Gespeg de wanderer et, de ce fait, ne leur octroient pas de territoire de réserve (Tribunal des revendications particulières, 2012). Cette décision constituait une négation de l'existence d'une communauté mi'gmaq sur la pointe de la Gaspésie, les excluant du territoire et du partage de ses richesses. Avec l'expansion de la population allochtone, on entend assimiler les Autochtones. Cela apparaît d'autant plus motivé que les grandes rivières à saumon sont convoitées par les pêcheurs sportifs et le bois, par les industriels. À cette époque, les Mi'gmaq acceptent des emplois saisonniers comme bûcherons et draveurs. Ils deviennent aussi manœuvres ainsi que serviteurs, mais tentent néanmoins de maintenir leur tradition de chasse et pêche (Mawiomi de Gespe'gawa'gi, 2018).

Au début du 20e siècle, c'est l'industrie papetière qui s'installe en Gaspésie. Le territoire des Mi'gmaq sera dorénavant exploité jusque dans son arrière-pays. La presque totalité des essences d'arbres dans toutes les classes de diamètre est dorénavant acheminée vers les usines. Cette époque accentue la pression vers la sédentarisation et l'assimilation. 
Pour les Mi'gmaq de Gespeg, la colonisation associée au défrichement, à l'exploitation industrielle des forêts et à la privatisation des rivières à saumon, ainsi que la négation généralisée de leurs droits par les autorités britanniques, puis canadiennes, marquent une période de survivance. Les Mi'gmaq voient leur environnement forestier être accaparé par les entreprises forestières, sans que leur existence ne soit prise en compte. Comme l'expriment Bouchard et Lévesque (2017, p. 207) au sujet des Innus, «... lorsque la forêt remplaça le castor dans les priorités économiques, le monde des Premières Nations s'effondra; les Indiens devinrent un problème, un obstacle au progrès, une question à résoudre». Ce verdict s'applique aussi aux Mi'gmaq de Gespeg.

Au Québec, les régimes forestiers n’ont jamais réellement eu parmi leurs objectifs de favoriser l'intégration des Autochtones et de leurs droits. Ils visent en priorité à assurer la gestion des volumes de bois requis pour approvisionner les usines (Chiasson et coll., 2005; Fréchette, 2009). Vécue à Gespeg, cette forme de gestion ne diffère pas de celle appliquée ailleurs au Canada (Wyatt, 2008).

\subsection{Une résurgence communautaire}

Cette ère de survivance se prolonge jusqu'au début des années 1970, période à partir de laquelle s'amorce la résurgence de la communauté de Gespeg. Au moment où les tribunaux canadiens reconnaissent l'existence de droits inhérents à des nations autochtones (Jugement Calder), plusieurs communautés entendent affirmer leur présence sur le territoire. C'est le cas à Gespeg. La reconnaissance gouvernementale de la communauté en 1973 et la constitution du conseil de bande de la Nation micmac de Gespeg marquent un jalon important de cette reconstruction. L'obtention de ce statut juridique, malgré des lacunes majeures, permet à la communauté de signifier son existence, de revendiquer des droits et d'obtenir des fonds gouvernementaux. L'officialisation du statut de la communauté dans le cadre légal canadien a mené à une certaine pérennisation de l'organisation sociale mi'gmaq. D'une communauté précarisée et en voie de dispersion depuis plus d'un siècle (Tribunal des revendications particulières, 2016), la communauté est passée en mode de revitalisation et a mis en ouvre des actions porteuses de possibilités pour son avenir (Nation micmac de Gespeg, 1999).

L'institutionnalisation de la Nation micmac de Gespeg permet l'acquisition d'un pouvoir de négociation avec les différents paliers gouvernementaux. Cela résout aussi le problème de l'«invisibilité» de la communauté sur la place publique. Cette reconnaissance limitée, qui exclut plusieurs aspects majeurs dont l'accès au territoire, n'en constitue pas moins le début du réveil existentiel de la communauté de Gespeg. Elle offre une voie d'expression légale permettant désormais à la communauté de s'organiser et d'exercer une gouvernance mi'gmaq, bien entendu limitée par sa portée et son exercice, 
mais réelle. Cet "outil» issu d'une législation externe à Gespeg sera employé pour implanter des mécanismes et réaliser des actions vers l'obtention de pouvoirs accrus pour la communauté.

Cependant, l'absence d'une assise territoriale attribuée à Gespeg subsiste. En terres privées, le territoire ancestral mi'gmaq est la propriété presque exclusive d'allochtones, à l'exception des lots appartenant à des membres de la communauté. En terres publiques, il demeure la chasse gardée du gouvernement du Québec et de ses mandataires. Nous avons constaté que la gouvernance pratiquée sur le territoire forestier ancestral des Mi'gmaq est centralisée. Malgré les transformations observées au cours des dernières décennies au Québec, l'État conserve ses prérogatives en ce qui concerne la gestion des ressources naturelles, la disposition des infrastructures, ainsi que l'encadrement des activités récréotouristiques et de conservation (Teitelbaum, 2015).

Malgré l'entrée en vigueur en 2013 de la Loi sur l'aménagement durable du territoire forestier, la situation des Mi'gmaq face à leur territoire forestier n'a pas changé. La gouvernance forestière exercée par le gouvernement du Québec est toujours subordonnée aux besoins de l'industrie. Le but visé de "partager les responsabilités découlant du régime forestier entre l'État, des organismes régionaux, des communautés autochtones et des utilisateurs du territoire forestier» (Gouvernement du Québec, 2019, article 1. $3^{\circ}$ ) n'a donné que peu de résultats pour les Mi'gmaq de Gespeg.

Ainsi, la gouvernance du territoire forestier est somme toute encore dominée par une approche extractiviste qui accorde la préséance aux entreprises forestières et minières (Fournis et Fortin, 2016). Les autres ayants droit (pourvoiries, villégiateurs, zones d'exploitation contrôlées, communautés autochtones, etc.) sont encadrés par des processus conçus pour permettre le bon fonctionnement des usages privilégiés par l'État québécois. Exception à cette règle, la plupart des statuts octroyés à des fins de conservation ne sont pas subordonnés à ceux ayant des fins extractives. Il s'agit d'un phénomène en expansion depuis l'émergence du militantisme environnemental dans les années 1960 au Québec (Poirier et Savard, 2015). Sur le territoire ancestral de Gespeg, ce phénomène s'observe notamment par la création d'aires protégées, telles que le parc Forillon en 1970, la réserve naturelle de l'Île-Bonaventure en 1971 et la réserve écologique de la Grande-Rivière en 2001.

Le renforcement de la législation environnementale sur le territoire se déroule simultanément avec la résurgence des Mi'gmaq de Gespeg. En parallèle à cette montée des Premières Nations et de la conservation des milieux naturels, les années 1990 voient s'amorcer le déclin de l'industrie forestière en Gaspésie, tant pour les pâtes et papiers que le sciage (MFFP, 2017a). Les fermetures d'usines diminuent la pression sur la ressource 
ligneuse. Cette conjoncture permet d'envisager l'accès à la ressource forestière auparavant inaccessible. Une gestion forestière mi'gmaq devient alors une option considérée, selon les propos entendus, comme ayant un potentiel de faisabilité plus élevé.

\subsection{Aujourd'hui, une communauté sans territoire et «urbaine»}

Actuellement, le conseil de la Nation micmac de Gespeg représente la communauté mi'gmaq de Gespeg et est membre du Secrétariat Mi'gmawei Mawiomi (SMM), un conseil tribal des trois communautés mi'gmaq (Gesgapegiag, Gespeg et Listuguj) du Gespe'gewa'gi, le septième district du Mi'gma'gi (le territoire mi'gmaq), vivant au Québec. Leur territoire ancestral correspond à la pointe de la Gaspésie et Anticosti ${ }^{7}$. Sa population s'élève à environ 1000 membres.

La communauté se caractérise par une grande dispersion de ses membres. Environ le tiers des membres réside dans la région de la baie de Gaspé et à peu près la moitié de la communauté habite la région de Montréal, où elle possède d'ailleurs un point de ralliement, soit un centre socioculturel. La Nation micmac de Gespeg est ainsi une communauté autochtone plutôt urbaine, une situation qui découle probablement en partie de l'absence d'un territoire de réserve délimité.

Cette situation motive la volonté de la communauté à disposer d'un territoire forestier (Blouin et coll., 2020). Évidemment, le territoire forestier représente une opportunité de développement socioéconomique pour les Mi'gmaq, mais leurs attentes et leurs besoins vont bien au-delà. Il s'agit en fait de disposer d'un ancrage territorial pour la communauté et d'un lieu de transmission de la culture mi'gmaq ancestrale (Blouin et coll., 2020). Le territoire se veut aussi l'occasion d'incarner les valeurs mi'gmaq par des gestes et des décisions dans des domaines associés à la protection des milieux naturels, la gestion des ressources naturelles ou le récréotourisme (Lynch et coll., 2010).

\section{Gmu'ge'qagua : la foresterie mi'gmaq}

\subsection{Les débuts}

L'engagement initial des Mi'gmaq de Gespeg en foresterie coïncide avec la période d'effervescence de la foresterie communautaire au Québec dans les années 1990, marquée par le programme des Forêts habitées (Bissonnette et coll., ce numéro). À cette époque, le Programme de mise en valeur des ressources du milieu forestier du gouvernement québécois visait à soutenir «les communautés autochtones dans l'acquisition

7. L'île d'Anticosti fait aussi partie du territoire ancestral des Innus de la Basse-Côte-Nord. 
de connaissances sur le territoire et les ressources du milieu forestier ainsi que dans la préparation et la réalisation de projets de mise en valeur des ressources du milieu forestier»(MRN, 2002, p. 4). Ce programme finançait divers projets forestiers autochtones cadrant avec les orientations gouvernementales.

C'est ainsi que la communauté dépose, en 1999, un projet d'aménagement forestier intégré pour un territoire situé à l'ouest du parc national Forillon. Sous le leadership de la Nation micmac de Gespeg, ce projet de foresterie communautaire implique 22 organismes de la région comme partenaires et s'inscrit dans la logique gouvernementale en gouvernance forestière communautaire qui semblait alors vouloir se mettre en place au Québec.

Cependant, le projet ne recevra pas l'aval du ministère des Ressources naturelles. L'allocation des volumes de matière ligneuse à un bénéficiaire de contrat d'approvisionnement et d'aménagement forestier (CAAF) et le refus de ce dernier d'en céder une part pour le projet de foresterie communautaire rendaient impossible le démarrage de cette initiative régionale. L'usine de papier journal du bénéficiaire allait pourtant fermer moins d'un an plus tard. Le programme des Forêts habitées, quant à lui, ne sera pas reconduit à son échéance et le projet ne fera plus partie des priorités du nouveau conseil de bande élu en 1999, qui optera pour une approche sectorielle plutôt que l'approche multifonctionnelle du projet précédent.

La Nation micmac de Gespeg établit alors un partenariat d'affaires avec des investisseurs privés, incluant la Ville de Gaspé, pour développer un projet d'usine de transformation du bois. Ce projet obtient le soutien des ministres délégués aux Affaires autochtones et au Développement régional, mais pas celui du ministre des Ressources naturelles. Selon les propos recueillis dans la communauté, le volume de bois nécessaire a été alloué à une entreprise compétitrice, qui l'a utilisé localement quelque temps avant de le transférer à son usine de la région voisine, annihilant ainsi le projet des Mi'gmaq de Gespeg.

En plus des projets forestiers, on assiste en 2000 à la création de la division foresterie, la Gmu'ge'qugua, et à l'embauche de personnel, ce qui marque la formalisation et le développement des initiatives forestières par l'intégration de ressources et de compétences professionnelles en foresterie au sein du conseil de bande. Un technicien forestier, membre de la communauté, est le premier employé embauché, grâce au financement de programmes gouvernementaux en foresterie autochtone. 
La première mission de la Gmu'ge'qagua consiste à assurer l'approvisionnement du site d'interprétation et de sa boutique. Il s'agit d'identifier des peuplements propices à l'approvisionnement en matériaux traditionnels, telles les frênaies noires, puis de développer et d'appliquer des interventions sylvicoles pour assurer leur pérennité et la qualité des matériaux traditionnels. Cette phase du développement de la foresterie a une orientation plutôt identitaire et culturelle, même si le prélèvement de matériaux traditionnels est effectué avec un objectif de vente d'artisanat. En effet, les retombées financières sont plutôt limitées comparativement à l'investissement nécessaire à son fonctionnement.

Dès 2002, l'étude du potentiel de mise en valeur des terres publiques intramunicipales ${ }^{8}$ (TPI) du territoire de la Municipalité régionale de comté (MRC) de La Côte-de-Gaspé pour la communauté s'ajoute à la mission. En effet, le conseil de la Nation micmac de Gespeg souhaite reprendre la gestion forestière de ces terres jusqu'alors effectuée par la MRC en vertu d'une entente avec le gouvernement du Québec. De son côté, la MRC juge la gestion des TPI inintéressante et déficitaire, étant donné leur potentiel ligneux limité, la faiblesse du marché du bois et les exigences administratives du gouvernement. Pour les Mi'gmaq, l'angle d'analyse ne se limite pas à l'aspect financier, car la communauté poursuit d'autres objectifs. De plus, des fonds gouvernementaux dédiés au développement forestier autochtone sont accessibles en support à l'atteinte de ces objectifs.

Les TPI offraient l'opportunité de développer une foresterie à petite échelle répondant à des besoins primaires (bois de chauffage) et traditionnels (matériaux pour l'artisanat), ainsi qu'aux aspirations territoriales de la communauté de Gespeg. Il s'agissait d'entamer un projet de foresterie fondée sur des valeurs identitaires, culturelles et communautaires, à partir d'un territoire forestier sur lequel la Gmu'ge'qagua intervient pour générer des retombées socioéconomiques.

\subsection{L'accélération}

En 2004, un autre technicien forestier, membre de la communauté, est embauché pour appuyer le processus de négociation et de signature (2005) de l'entente de délégation de gestion des TPI. La Gmu'ge'qagua entreprend de plus la conception d'un plan de développement forestier pour la communauté (Nation micmac de Gespeg, 2005) qui a pour objectifs de documenter le profil socioéconomique de la communauté, d'identifier ses besoins, de présenter des projets répondant à ceux-ci, et de définir des priorités d'action. L'élaboration du plan de développement est inspirée par les documents d'orientation de l'Assemblée des Premières Nations du Québec et du Labrador concernant les consultations et le développement durable (Nation micmac de Gespeg, 2005).

8. Les TPI sont des terres publiques comprises dans les limites municipales et propriété du gouvernement du Québec. 
Cette réflexion est alors conduite par la Gmu'ge'qagua, en collaboration avec le conseil de bande, et alimentée par une consultation non probabiliste des membres réalisée principalement par téléphone.

Les besoins identifiés sont: 1) les besoins communautaires; 2) les besoins de représentation et de valorisation du territoire; et 3) les besoins de développement économique. Une orientation générale émerge de cet exercice, soit: "Le principal souci des membres est d'assurer la survie de notre forêt et de notre culture» (Nation micmac de Gespeg, 2005, p. 20).

Les besoins communautaires énoncés sont variés, et vont de l'amélioration de l'accès au territoire et à ses ressources à la conservation de la culture mi'gmaq. Les besoins de représentation de la communauté et de valorisation du territoire correspondent à une volonté de participer à la gestion forestière avec un regard territorial, ainsi que d'assurer le respect des droits ancestraux. Les besoins économiques découlent de la préoccupation des membres de bénéficier des retombées de leur forêt. Pour ce faire, on veut renforcer l'expertise mi'gmaq en foresterie, créer des emplois et fournir des biens et services à la communauté (du bois de chauffage, par exemple). Les besoins ainsi identifiés ont servi de fondement à la définition des actions et des projets subséquents, enrichissant du même coup le mandat de la Gmu'ge'qagua. Ces actions devaient respecter quatre principes directeurs: 1) le respect des droits et des besoins en tant que Première Nation; 2) la gestion intégrée du territoire et des ressources, en cogestion; 3) l'aménagement intégré des ressources; et 4) la certification environnementale autochtone (Nation micmac de Gespeg, 2005).

Le plan de développement forestier est un jalon important de la structuration des intentions en foresterie de la communauté. Ce document a permis de formaliser la vision et d'énoncer des actions. Les actions visent alors la cogestion des TPI, l'exploitation d'une érablière commerciale, l'aménagement des peuplements forestiers à des fins d'approvisionnement en matériaux traditionnels pour l'artisanat, la réalisation d'aménagements récréotouristiques et le développement d'entreprises forestières.

La signature d'une entente triennale de délégation de gestion forestière (2005) officialise la première implication d'envergure de Gespeg en foresterie. Elle s'applique aux TPI de la MRC de La Côte-de-Gaspé d'une superficie de 2650 hectares. Ces terres sont fortement morcelées et dispersées, ce qui représente une contrainte pour la production de bois. Mais pour l'approvisionnement en plantes et en matériaux destinés à l'artisanat, cette dispersion offre une diversité intéressante. Avant la signature, la communauté 
de Gespeg avait déterminé que cette entente ne visait pas prioritairement un objectif de rentabilité financière, mais qu'elle servait plutôt des intérêts communautaires, territoriaux et économiques (Nation micmac de Gespeg, 2005).

La mise en valeur des TPI exigera la création d'une entreprise de vente de bois de chauffage pour éponger les frais de gestion, de planification et de suivi, que les subventions n'auront pas suffi à couvrir. Le gouvernement du Québec impose un encadrement strict des gestionnaires de TPI, appliquant des exigences semblables aux unités d'aménagement forestier (UAF) en termes de planification et de consultation. Ces exigences sont jugées disproportionnées et limitatives par les intervenants mi'gmaq interrogés, compte tenu de la superficie réduite des TPI et de leur potentiel de revenu restreint.

Le démarrage de l'entreprise a néanmoins permis de créer quelques emplois et de diversifier l'expertise en gestion de la Nation, jusqu'alors cantonnée aux pêcheries, au tourisme culturel et à l'artisanat. Grâce aux revenus générés par la vente de bois de chauffage aux non-membres, du bois de chauffage a été distribué gratuitement aux aînés et vendu au prix coûtant aux membres de la communauté.

\subsection{Consolidation}

Au début des années 2010, la Nation micmac de Gespeg obtient des attributions annuelles de matière ligneuse, soit $5000 \mathrm{~m}^{3}$ en bois de sciage et $2000 \mathrm{~m}^{3}$ en bois de chauffage, sous forme de permis de récolte en terres publiques. Ces allocations ont permis l'essor de l'entreprise de bois de chauffage.

Le volume en bois de sciage est alors récolté par une entreprise forestière disposant d'une scierie, selon les modalités d'une entente avec la communauté. Des échanges de bois sont effectués lorsque du bois de sciage se retrouve sur les parterres de coupe de bois de chauffage ou à l'inverse. Selon les commentaires recueillis, cette formule offre l'opportunité de maximiser les retombées pour les partenaires en fonction de leurs intérêts commerciaux respectifs.

Depuis 2014, les TPI sont certifiés selon le standard du Forest Stewardship Council (FSC). Le certificat est détenu par le Syndicat des producteurs de bois de la Gaspésie, dont la Nation micmac de Gespeg est membre. Celle-ci est aussi une partie prenante consultée pour l'obtention de la certification FSC sur les terres publiques. Elle a ainsi participé à l'identification des forêts de haute valeur pour la conservation (FHVC) dans le cadre du processus de certification des territoires publics de la Gaspésie en 2015 
(CGCCÎM, 2015). De même, elle s'est impliquée dans le comité régional ayant pour mandat l'étude de scénarios d'établissement d'aires protégées terrestres pour la Gaspésie (CRÉGîM, 2014).

Ces processus de consultation s'ajoutent à celui du ministère des Forêts, de la Faune et des Parcs du Québec (MFFP). Avant l'entrée en vigueur de la Loi sur l'aménagement durable du territoire forestier en 2013, les entreprises détentrices de CAAF effectuaient un processus d'harmonisation. Selon les informations obtenues, les consultations avec la Nation micmac de Gespeg étaient alors pratiquement inexistantes. Subséquemment, la participation aux tables locales de gestion intégrée des ressources et du territoire 9 (TLGIRT) a été plutôt difficile selon ces mêmes informateurs.

En 2014, le MFFP et le SMM «Convenaient d'un processus de participation à la planification forestière adapté aux besoins des Mi'gmaq" (MFFP, 2017b, p. 3). Ce processus distinct a été mis en ouvre "afin de connaître et d'intégrer les valeurs et les besoins des communautés micmaques» (MFFP, 2017b). C'est le Secrétariat Mi'gmawei Mawiomi (SMM) qui est mandaté pour y représenter les trois communautés mi'gmaq de la Gaspésie. Une fiche, nommée "Enjeu valeurs autochtones", est le produit de ce processus pour lequel elle sert de repère (ibid.). Cette fiche identifie des valeurs mi'gmaq au sujet de peuplements forestiers ayant une valeur culturelle, de la protection de l'habitat du saumon et des conditions de pratique des activités traditionnelles en forêt, plus particulièrement la chasse. Des indicateurs avec des cibles y sont définis en vue d'être intégrés à la planification forestière gouvernementale (ibid.).

\subsection{La réorientation}

Le projet d'exploitation d'une érablière sucrière en cours de réalisation est le dernier volet de ce cheminement. Ce projet, qui comprendra 8000 entailles à sa mise en opération en 2020, permettra de concrétiser une action prévue depuis 2005 au plan de développement forestier de la communauté. Nous avons constaté que ce plan a joué un rôle majeur dans la structuration de l'implication des Mi'gmaq de Gespeg en foresterie au cours des 15 dernières années, en définissant les jalons du parcours forestier de la communauté.

Ces engagements en foresterie ne semblent toutefois pas combler de manière acceptable les attentes et les besoins de la communauté (Blouin et coll., 2020). C'est pourquoi la Nation micmac de Gespeg souhaite réaliser un projet de forêt communautaire. La forêt communautaire est une entité dotée d'un mandat explicite et d'une autorité

9. Les TLGIRT sont un mécanisme de consultation mis en œuvre dans le cadre de la planification forestière des terres publiques au Québec. 
légale pour gérer un territoire forestier au bénéfice d'une communauté locale (Krogman et Beckley, 2002), ce qui permet la construction d'un espace politique de contrôle, de décision et de distribution de ses bénéfices (Bullock et Hanna, 2012).

Ce projet a fait l'objet d'un processus de co-construction avec le premier auteur, incluant des consultations et des analyses. Ce processus a mené à la définition d'un concept préliminaire de forêt communautaire et a comporté des démarches auprès des autorités et de partenaires potentiels. Il est encore en cours.

\section{Gmu'ge'qagua, l'institution forestière plaçant Gespeg au cœur d'une gouvernance renouvelée}

Notre recherche donne à penser que la Nation micmac de Gespeg et sa Gmu'ge'qagua ont fait le tour des possibilités offertes aux communautés autochtones par le régime forestier québécois. Avec leur projet de forêt communautaire, les Mi'gmaq de Gespeg confrontent ce régime avec des demandes constructives, mais qui déstabilisent le gouvernement du Québec par le changement de paradigme qu'elle suppose.

Les ministères contactés ont eu des postures différentes selon que leur rôle était remis en question ou qu'ils y voyaient une ouverture pour réaliser leur mission. Ces réactions concordent avec la notion de gouvernementalité (Arts, 2014). Ce concept s'appuie sur la notion que l'État n'accepte que des changements qui ne minent pas sa raison d'être. Le projet de gestion multiressource confronte les autorités du MFFP dans leur rôle de gestionnaire principal et dans leurs objectifs centrés sur la matière ligneuse.

La vision et les objectifs du projet de forêt communautaire se fondent sur le territoire en tant que lieu d'incarnation de l'identité mi'gmaq, milieu de vie multidimensionnel et support aux aspirations de la communauté. L'extraction de la matière ligneuse n'est qu'un aspect des possibilités offertes par la forêt aux Mi'gmaq. Cela rejoint la foresterie pratiquée actuellement par les Mi'gmaq de Gespeg, qui vise à assurer l'accès à la forêt tout en satisfaisant des besoins locaux en bois comme en matériaux traditionnels pour l'artisanat. L'existence de la Gmu'ge'qagua ne repose pas sur une volonté d'extraction de la matière ligneuse. Elle se veut un moyen d'atteindre des objectifs communautaires.

L'industrie forestière est perçue par nos informateurs comme un secteur économique ne permettant pas de générer des revenus prévisibles pour des risques modérés. De même, parmi les activités dans lesquelles la Nation micmac de Gespeg s'investit déjà, la foresterie génère difficilement des profits, comparativement aux pêcheries ou à l'énergie 
éolienne (Blouin et coll., 2020). Il faut dire aussi que la pointe de la Gaspésie est considérée en marge du marché du bois en raison de l'absence de grands investisseurs, du déclin régional de l'industrie du bois, de la perte d'infrastructures et des épidémies récurrentes de la tordeuse des bourgeons de l'épinette. Cette dynamique défavorable à l'industrie forestière représente en contrepartie une occasion de collaboration entre autochtones et allochtones afin de concevoir un développement régional différent.

En effet, ce contexte politico-économique crée possiblement des circonstances favorables pour engendrer un moment transformateur (Peluso et Lund, 2011). Cette conjoncture changeante est propice pour envisager d'autres options d'allocation du territoire forestier et de ses ressources (Ribot et Peluso, 2003). Selon les propos recueillis, les Mi'gmaq et leurs partenaires régionaux semblent discerner ce moment où un projet innovant de gouvernance forestière devient possible.

Cette collaboration régionale au projet est jugée essentielle par le Conseil de la Nation micmac de Gespeg, dont la position rejoint la description que faisait la Nation en 1999 des relations intra-régionales en ces termes: "...les membres vivent en étroite relation avec la population non-autochtone, que cela soit par le biais du travail, des loisirs ou autres. De plus, des liens d'amitié sont également créés à partir de ces relations. Nous sommes intégrés à la vie régionale et sommes comptés comme résidants de nos municipalités respectives.» (Nation micmac de Gespeg, 1999, sans pagination). Cette dynamique particulière à la région s'exprime aussi dans plusieurs collaborations en tourisme et au niveau économique. Mais surtout, il semble exister une volonté de réconciliation concrète de part et d'autre, comme l'illustre la présence d'une délégation commune de la Nation micmac de Gespeg, de la Ville de Gaspé et de la Ville de Percé au Sommet de la réconciliation avec les peuples autochtones tenu à l'été 2018 à Montréal.

Le projet de forêt communautaire se heurte toutefois à une situation de blocage institutionnel qui empêche sa concrétisation. L'implication mi'gmaq en foresterie semble avoir atteint ses limites. Par exemple, l'entente de délégation forestière pour les TPI n'offre pas la latitude nécessaire pour développer une façon d'habiter et d'aménager le territoire qui réponde à leurs attentes. L'accès au territoire et à la ressource demeure fortement contrôlé et encadré par le gouvernement du Québec. Cette entente ainsi que les permis et les autres ententes forestières représentent des occasions de développer la Gmu'ge'qagua. Ils semblent servir davantage de passage transformatif vers un projet de gouvernance territoriale et forestière, comme celui de forêt communautaire initialement tenté à la fin des années 1990, que constituer une fin satisfaisante en soi. 
Les refus des détenteurs de droits sur la ressource forestière, soit le gouvernement et le bénéficiaire de CAAF de l'époque, face aux projets de forêt communautaire puis de co-entreprise de transformation du bois, ont mené à un repositionnement de la Nation micmac de Gespeg vers des engagements en foresterie correspondant au cadre gouvernemental. Ces implications plus restreintes en termes de droits sur le territoire et la ressource ont probablement atteint leurs limites. Elles sont maintenant parfois questionnées dans la communauté en raison du plafonnement de leurs retombées.

Envisagés comme un tremplin vers une forêt communautaire, les engagements en foresterie des 15 dernières années sont décevants somme toute pour la Nation micmac de Gespeg, en particulier - comme on nous l'a dit - si on les compare aux résultats de leurs engagements dans les pêcheries et l'énergie éolienne. Les exigences et les procédures administratives sont lourdes et coûteuses pour des retombées en deçà des attentes. Le conseil et des membres de la communauté se questionnent ainsi sur la pertinence de s'investir dans l'exploitation forestière, si cela fait courir le risque de pertes financières sans bénéfices significatifs en retour. L'échec de la deuxième tentative de mise en place d'une gouvernance territoriale et forestière mi'gmaq pourrait amener la Nation micmac de Gespeg à changer de stratégie pour obtenir un territoire forestier.

En somme, de l'avis de nos informateurs, le cadre gouvernemental actuel en foresterie autochtone au Québec demeure limité dans ses possibilités. Mais qu'en est-il alors de la notion de forêt de proximité (Bissonnette et coll., ce numéro)? Ce concept fait référence à l'instauration d'une politique de foresterie communautaire explicitement reconnue juridiquement, mais sans obligation de résultat. En effet, le régime actuel se montre favorable à l'établissement d'une gouvernance communautaire dans laquelle un organisme du milieu assure une fonction déterminante (MFFP, 2005). Toutefois, les modalités d'application de la forêt de proximité restent alignées sur les orientations conventionnelles de la politique forestière québécoise, et les activités déléguées ainsi que les responsabilités afférentes sont limitées à l'exploitation et à la planification forestière dans le respect du cadre gouvernemental (MFFP, 2015). Les communautés autochtones intéressées, comme Gespeg, continuent d'attendre un dénouement à ce sujet, encore incertain.

Pour les Mi'gmaq, les ententes de "sous-traitance» conclues avec le gouvernement du Québec ne correspondent pas à leur définition de la cogestion forestière. Pour eux, une réelle cogestion signifie un partage des pouvoirs et des responsabilités déterminé à partir de l'établissement d'une vision commune. Cette appréciation ne semble pas exceptionnelle chez les communautés autochtones en matière de cogestion (Berkes et coll., 1991). Pour les informateurs, la cogestion est vue comme une option intérimaire 
vers une gouvernance forestière et territoriale mi'gmaq. Le modèle de gouvernance forestière mi'gmaq partenariale et régionale qu'ils souhaitent mettre en ouvre correspond à leur conception d'une cogestion effective.

Comparativement aux communautés allochtones, il existe peut-être des possibilités de transformation du cadre politique plus grandes pour les communautés autochtones, compte tenu de l'évolution du droit et de la reconnaissance des droits ancestraux des Premières Nations. Les droits constitutionnels autochtones sont susceptibles de forcer une ouverture du gouvernement du Québec face aux revendications identitaires et territoriales (Grammond, 2009), comme celle que pourrait rencontrer l'instauration de forêts communautaires. Il s'agit de la seule condition actionnable, identifiée à la figure 3 sous le vocable "Politiques (buts et outils)», qui n'a pas évolué significativement dans les dernières décennies.

\section{Figure 3 : Évolution des conditions actionnables de la gouvernance du territoire forestier des Mi'gmaq de Gespeg de 1995 à 2020.}

Conditions « actionnables » en 1995

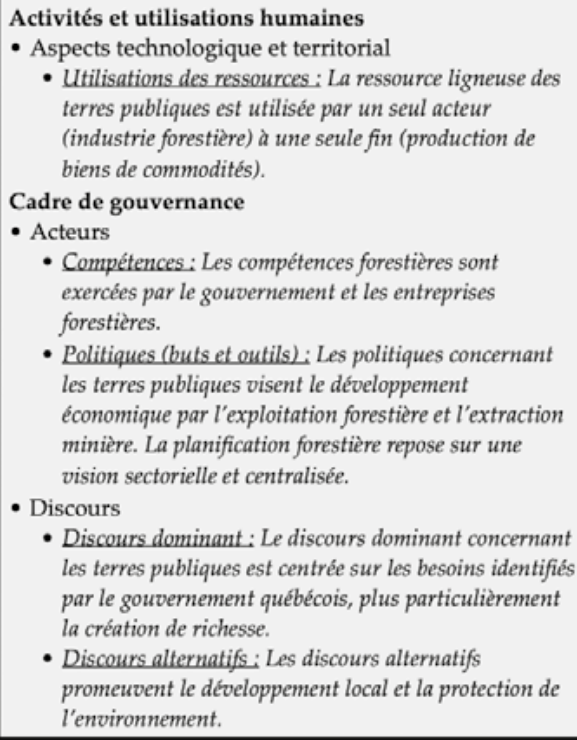

- Discours dominant: Le discours dominant concernant les terres publiques est centrée sur les besoins identifiés par le gouvernement québécois, plus particulièrement la création de richesse.

- Discours alternatifs: Les discours alternatifs promeuvent le developpement local et la protection de l'environnement.

Conditions « actionnables " en 2020

\begin{tabular}{|l|l|}
\hline Activités et utilisations humaines \\
- Aspects technologique et territorial \\
- Utilisations des ressources: La ressource ligneuse des \\
terres publiques demeure majoritairement utilisée par \\
un seul acteur (industrie forestière) à une seule fin \\
(production de biens de commodités). Les Mi'gmaq \\
utilisent une partie de celle-ci à des fins culturelles \\
(matériaux d'artisanat) et pour combler des besoins \\
locaux (bois de chauffage). \\
Cadre de gouvernance \\
- Acteurs \\
- Compétences: Les compétences forestières sont \\
exercées par le gouvernement et les entreprises \\
forestières. Les Mi'gmaq exercent des compétences \\
forestières en cogestion avec le gouvernement. \\
- Politiques (buts et outils): Les politiques concernant \\
les terres publiques visent le développement \\
économique par l'exploitation forestière et l'extraction \\
minière. La planification forestière repose sur une \\
vision sectorielle et centralisée, incluant un processus \\
consultatif auprès des Mi'gmaq. \\
- Discours \\
- Discours dominant: Le discours dominant concernant \\
les terres publiques est centrée sur les besoins identifiés \\
par le gouvernement québécois, plus particulièrement \\
la création de richesse. \\
- Discours alternatifs: Les discours alternatifs \\
promeuvent le développement local, la protection de \\
l'environnement et les droits autochtones. Les \\
Mi'gmaq possèdent un discours forestier pour \\
structurer leurs actions et influer sur le devenir de leur \\
territoire forestier. \\
\hline
\end{tabular}

Cette transformation des buts de l'aménagement des terres publiques est nécessaire pour permettre une nouvelle gouvernance forestière du territoire ancestral des Mi'gmaq de Gespeg. Les autres conditions actionnables ont toutes connu des avancées. 
L'utilisation des ressources et du territoire est maintenant possible dans une certaine mesure. La communauté s'est dotée d'une expertise et de ressources professionnelles avec la Qmu'ge'qagua. Elle s'est aussi construit un discours alternatif face au discours dominant, tel qu'illustré par son plan de développement et son projet de forêt communautaire. De même, le discours dominant s'est transformé et les Autochtones sont maintenant inclus dans la vision officielle portée par le régime forestier québécois, comme par exemple dans la Stratégie d'aménagement durable des forêts (MFFP, 2005). La condition "Politiques» s'est aussi transformée en termes d'outils par l'intégration de facto des Autochtones dans des mécanismes procéduraux spécifiques de consultation et des programmes dédiés. Cependant, les politiques forestières demeurent structurées autour d'une vision de gouvernance centralisée et sectorielle, qui confronte les attentes autochtones face au territoire forestier (Fuss et coll., 2018). Elles se distinguent de la gouvernance forestière communautaire et territoriale à laquelle aspirent les Mig'maq de Gespeg (Blouin et coll., 2020). En termes de gouvernance, la foresterie pourrait bien n'être qu'une activité complémentaire dans la perspective holistique d'un territoire forestier mi'gmaq.

Depuis l'échec de la première tentative de forêt communautaire, la démarche des Mi'gmaq en foresterie semble s'inscrire dans une stratégie progressive de construction de leurs compétences et de leur crédibilité, tout en gardant le cap sur la concrétisation de leur vision première. Notre recherche nous amène à concevoir une démarche comprenant sept étapes que nous synthétisons ainsi : 1) une problématique vécue et subie, liée à la longue histoire de colonisation, de dépossession et d'assimilation; 2) un constat, une compréhension (une internalisation) de la problématique menant à une vision souhaitée; 3) une volonté et une possibilité d'agir; 4) une évaluation des capacités et des possibilités; 5) une prise de décision et un plan d'action (une stratégie); 6) une évaluation des objectifs atteints; et 7) un réajustement de la stratégie. Ce cheminement de la Nation micmac de Gespeg et ses choix stratégiques concomitants ont plusieurs points en commun avec le vécu des Mi'gmaq de Listuguj (Wyatt et coll., 2019), mais sans événements conflictuels de grande ampleur. Le contexte de cohabitation dans lequel vivent les Mi'gmaq de Gespeg est une explication probable de ce fait. Les propos que nous avons recueillis convergent vers une volonté de maintenir et de renforcer une cohabitation positive entre communautés dans la région de Gaspé.

Les Mi'gmaq de Gespeg ont agi tel un entrepreneur institutionnel efficace au cours des quinze dernières années, en ciblant les conditions sur lesquelles ils pouvaient exercer une influence pour induire un mouvement vers la gouvernance forestière souhaitée. Par leur intégration au système forestier, les Mi'gmaq ont développé une compréhension du système de gouvernance en place qui leur a permis d'y déceler les occasions de changement (Westley et coll., 2013). Cette stratégie graduelle de changements 
cumulatifs et successifs des Mig'maq s'oriente maintenant vers la définition d'une vision commune avec des partenaires locaux. Les Mi'gmaq y jouent un rôle de leader dans la transformation de l'autorité exercée sur les ressources et le territoire régional. Les acquis de leurs actions permettent d'envisager une transformation du cadre politique intégrant les objectifs locaux par l'expérimentation d'une forme de gouvernance fondée sur un paradigme territorial et communautaire. Ce cheminement se compare à celui identifié dans la théorie sur l'agentivité transformative dans les systèmes socio-écologiques (Westley et coll., 2013) comme favorisant la réussite des entrepreneurs institutionnels.

\section{Conclusion}

Les Mi'gmaq de Gespeg se sont engagés sur la voie de l'autonomie gouvernementale au cours des dernières décennies. En foresterie, cette démarche a pris plusieurs formes (entente de cogestion, droits d'exploitation, consultation, partenariat). Ces engagements mi'gmaq basés sur la construction progressive de l'expertise, l'expansion du domaine d'intervention et la collaboration avec les intervenants régionaux et forestiers semblent avoir donné leur mesure sans atteindre la satisfaction de leurs attentes. Depuis la fin des années 1990, les Mi'gmaq de Gespeg souhaitent assurer la gouvernance d'un territoire forestier en collaboration avec des partenaires régionaux. Un tel projet reste en rade. Cette situation appelle une question majeure. En contraste avec la Paix des Braves, l'État québécois est-il prêt à transformer sa pratique de la cogestion forestière avec les Premières Nations, particulièrement dans le sud du Québec en contexte plus densément peuplé?

Dans le cadre du régime actuel, la disposition sur la forêt de proximité pourrait rendre la chose possible (Gouvernement du Québec, 2019). Toutefois, l'environnement politique devra changer, car le gouvernement du Québec ne montre pour l'instant aucun empressement à concrétiser ses intentions à l'égard des forêts communautaires. Les avancées en droit autochtone conjuguées à la mise en application effective de cette disposition permettent d'envisager le dépassement de la relation de sous-traitance privilégiée par les autorités québécoises et d'asseoir une gouvernance où des instances autochtones tiendraient un rôle déterminant. 


\section{Bibliographie}

Arts, B. (2014). Assessing forest governance from a 'Triple G' perspective: Government, governance, governmentality. Forest Policy and Economics, (49): 17-22.

Arts, B., Visseren-Hamakers, I.J. (2012). Forest governance: A state of the art review. European Tropical Forest Research Network News, (53): avril 2012.

Asselin, H., Basile, S. (2018). Concrete ways to decolonize research. ACME: An International Journal for Critical Geographies, 17(3), 643-650.

Beaudoin, J.-M., St-Georges, G., Wyatt, S. (2012). Valeurs autochtones et modèles forestiers: Le cas de la Première Nation des Innus d'Essipit. Recherches Amérindiennes au Québec, 42(2-3), 97-109.

Beaudoin, J.-M., Bouthillier, L., Bulkan, J., Nelson, H., Trosper, R., Wyatt, S. (2016). What does "First Nation deep roots in the forests" mean? Identification of principles and objectives for promoting forest-based development. Canadian Journal of Forest Research, 46, 508-519.

Berkes, F., George, P., Preston, R. J. (1991). Co-management, the evolution in the theory and practice of the joint administration of living resources. Alternatives, 18(2), 12-17.

Berkes, F. (2009). Evolution of co-management: Role of knowledge generation, bridging organizations and social learning. Journal of Environmental Management, (90), 1692-1702.

Binder, C. R., Hinkel, J., Bots, P. W. G., Pahl-Wostl, C. (2013). Comparison of Frameworks for Analyzing Social-ecological Systems. Ecology and Society, 18(4), 26.

Bissonnette, J-F., Blouin, D., Teitelbaum, S., Bouthillier, L. (2020). Vers des forêts de proximité en terres publiques? Valoriser les approches de cogestion en foresterie communautaire au Québec. Gouvernance (ce numéro: détails à venir)

Blouin, D., Bissonnette, J-F., Bouthillier, L. (2020). Habiter son territoire forestier: valeurs, attentes et vision des Mi'gmaq de Gespeg. Recherches Amérindiennes au Québec, Accepté, numéro à paraître.

Bouchard, S., Lévesque, M-C. (2017). Le peuple rieur, hommage à mes amis innus. Montréal: Lux. 316p.

Bourassa, M., Bélair, L., Chevalier, J. (2007). Les outils de la recherche participative. Éducation et francophonie, $X X X V(2), 1-11$.

Brusati, L., Iacuzzi, S., Fedele, P., Ianniellio, M. (2016). Governing the Global Commons: how can SMEs be Engaged? International OFEL Conference on Governance, Management and Entrepreneurship, 1-19.

Bullock, R., Hanna, K.S. (2012). Community forestry: local values, conflict and forest governance. Cambridge: Cambridge University Press. 186 p.

CGCCÎM. (2015). Rapport final du Comité sur les aires protégées terrestres de la Gaspésie. Conférence régionale des élus Gaspésie-Îles-de-la-Madeleine, 168p.

Chiasson, G., Boucher, J. L., Thibault, M. (2005). La forêt plurielle: nouveau mode de gestion et d'utilisation de la forêt, le cas de la Forêt de l'Aigle. VertigO, 6(2), 1-11.

Clement, F. (2010). Analysing decentralised natural resource governance: proposition for a "politicised" institutional analysis and development framework. Policy Sciences, (43), 129-156. 
Cole, D. H., Epstein, G., McGinnis, M. D. (2019). Combining the IAD and the SES frameworks. International Journal of the Commons, (13), 1-32.

CRÉGÎM. (2014). Identification des Forêts de Haute Valeur pour la Conservation sur les unités d'aménagement de la Gaspésie 112.62 et 112.63. Corporation de gestion de la certification forestière des territoires publics de la Gaspésie-Îles-de-la-Madeleine, 97p.

Fortier, J-F., Wyatt, S. (2019). Émergence et évolution de la collaboration dans la planification forestière du Nitaskinan. Canadian Journal of Forest Research, (49), 350-360.

Foudriat, M., Donovan, P., Riviera, M-T. (2000). Les méthodologies participatives et la question du changement dans les contextes d'action: réflexion théorique et méthodologique à partir d'une comparaison d'expériences en Équateur, au Chili et en France. Revue canadienne d'études du développement, 21(1), 495-521.

Fournis, Y., Fortin, M-J. (2016). Les régimes de ressources au Canada: les trois crises de l'extractivisme. VertigO, 15(2), 2-13.

Fuss, G. E., Stennberg, J. W.N., Weber, M. L., Smith, M.A., Creed, I. F. (2018). Governance as a driver of change in the Canadian boreal zone. Dossiers environnement, DOI 10.1139/er-2018-0057.

Fréchette, A. (2009). La gouvernance forestière au Québec: le défi du changement institutionnel dans les systèmes socio-écologiques interdépendants. VertigO, (6).

Gouvernement du Québec. (2019). Loi sur l'aménagement durable du territoire forestier. Repéré à http://legisquebec.gouv.qc.ca/fr/ShowDoc/cs/A-18.1

Grammond, S. (2009). La gouvernance territoriale au Québec entre régionalisation et participation des peuples autochtones. Canadian journal of political science, 42(4), 939-956.

Hébert, M. (2006). De la consultation à la justice sociale: Les conditions de la diffusion d'une éthique autochtone en matière de gestion des ressources forestières. Recherches Amérindiennes au Québec, 36(2-3), 33-42.

Krogman, N., Beckley., T. (2002). Corporate "Bail-Outs" and Local "Buyouts": Pathways to Community Forestry? Society and Natural Ressources, 15, 109-127.

Levy, D., Scully, M. (2007). The Institutional Entrepreneur as Modern Prince: The Strategic Face-off Power in Contested Field. Organization Studies, 28(07), 971-991.

Lynch, M-F., Duinker, P., Sheehan, L., Chute, J. (2010). Sustainable Mi'kmaw cultural tourism development in Nova Scotia, Canada: examining cultural tourist and Mi'kmaw perspectives. Journal of Sustainable Tourism, 18(4), 539-556.

Mawiomi de Gespe'gawa'gi. (2018). Nta'tugwaqanminen, Notre histoire: L'évolution des Mi'gmaqs de Gespe'gewa'gi. Halifax et Winnipeg: Presses de l'Université d'Ottawa et Fernwood Publishing, 297p.

MFFP. (2005). Stratégie d'aménagement durable des forêts. Ministère des Forêts, de la Faune et des Parcs du Québec, 52p.

MFFP. (2015). Forêts de proximité Orientations ministérielles sur la mise en ouvre. Ministère des Forêts, de la Faune et des Parcs du Québec, 9p. 
MFFP. (2017a). Plan d'aménagement forestier intégré tactique 2018-2023, unité d'aménagement 112-62, région de la Gaspésie-Îles-de-la-Madeleine. Ministère des Forêts, de la Faune et des Parcs du Québec, 93p.

MFFP. (2017b). Fiche enjeu valeurs autochtones. Ministère des Forêts, de la Faune et des Parcs du Québec, fiche R11-T-18-26, 14p.

Moisdon, J-C. (1984). Recherche en gestion et intervention. Revue française de gestion, 47-48, 21-39.

MRN. (2002). Programme de mise en valeur des ressources du milieu forestier, description et modalités d'application. Ministère des Ressources naturelles du Québec, 10p.

Myers, R., Hansen, C. P. (2019). Revisiting A Theory of Access: A review. Society and Natural Resources, DOI 10.1080/08941920.2018.1560522.

Nation micmac de Gespeg. (1999). La présence des Micmacs en Gaspésie. Mémoire présenté à la Commission des lieux et monuments historiques du Canada, 97p.

Nation micmac de Gespeg. (2005). Plan de développement forestier. 42p.

Ostrom, E. (1990). Governing the Commons: The Evolution of Institutions for Collective Action. New York: Cambridge University Press. 280p.

Ostrom, E. (2007). A diagnostic approach for going beyond panaceas. Proceedings of the National Academy of Sciences of the United States of America, 1004(34), 15181-15187.

Peluso, N. L., Lund, C. (2011). New frontiers of land control: Introduction. The Journal of Peasant Studies, 38(4), 667-681.

Pinna, S., Malenfant, A., Hébert, B., Côté, M. (2009). Portrait forestier historique de la Gaspésie. Gaspé: Consortium en foresterie Gaspésie-Les-Îles. 204p.

Poirier, V., Savard, S. (2015). Présentation: le militantisme environnemental au Québec, ou comment l'environnement est devenu un enjeu politique. Bulletin d'bistoire politique, 23(2), 15-31.

Ribot, J. C., Peluso, N. L. (2003). A Theory of Access. Rural Sociology, 68(2), 153-181.

Roy, S. N. (2016). Chapitre 8: L'étude de cas. Recherche sociale. (6e éd.) Québec: Presses de l'Université du Québec.

Teitelbaum, S. (2015). Le respect des droits des peuples autochtones dans le régime forestier québécois: quelle évolution (1960-2014)? Recherches sociographiques, 56(2-3), 299-323.

Teitelbaum, S., Montpetit, A., Bissonnette, J-F., Chion, C., Chiasson, G., Doyon, F., ... (2019). Studying Resource-Dependent Communities Through a Social-Ecological Lens? Examining Complementarity with Existing Research Traditions in Canada. Society and Natural Resources, 32(1), 93-112.

Tremblay, D-G., Demers, G. (2018). Les recherches partenariales et collaboratives: peut-on simultanément théoriser et agir? Recherches sociographiques, LIX(1-2), 99-119.

Tribunal des revendications particulières. (2012). La Nation micmac de Gespeg c. Sa Majesté la Reine du chef du Canada, Déclaration de revendication aux termes de la règle 41 des Règles de procédure du Tribunal des revendications particulières. Dossier: SCT-2001-12. 
Tribunal des revendications particulières. (2016). La Nation micmac de Gespeg c. Sa Majesté la Reine du chef du Canada. Dossier: SCT-2001-12.

Westley, F. R., Tjornbo, O., Schultz, L., Olsson, P., Folke, C., Crona, B., Bodin, O. (2013). A Theory of Transformative Agency in Linked Social-Ecological Systems. Ecology and Society, 10(3), 27. DOI 10.5751/ES-05072-180327.

Whaley, L., Weatherhead, E. K. (2014). An Integrated Approach to Analyzing (Adaptive) Comanagement Using the "Politicized" IAD Framework. Ecology and Society 19(1), 10.

Wyatt, S. (2008). First Nations, forest lands, and "aboriginal forestry" in Canada: from exclusion to comanagement and beyond. Canadian Journal of Forest Research, 38, 171-180.

Wyatt, S., Fortier, J-F., Hébert, M. (2010). Collaboration entre Autochtones et autres acteurs forestiers du Québec: portrait d'une diversité de pratiques et de modèles. The Forestry Chronicle, 86(2), 243-255.

Wyatt, S., Hébert, M., Fortier, J-F., Blanchet, É-J., Lewis, N. (2019). Strategic approaches to Indigenous engagement in natural resource management: use of collaboration and conflict to expand negotiating space by three Indigenous nations in Quebec, Canada. Canadian Journal of Forest Research, 49, 375-386.

Yin, R. K. (2014) Case study research and applications: Design and methods. Sage publications. 282p.

\section{Remerciements}

Cette recherche a été possible grâce à la collaboration et au partenariat avec la communauté mi'gmaq de Gespeg. Son appui a été essentiel à la réalisation du projet de recherche ayant permis la rédaction de cet article. Nous remercions les membres, ainsi que le Conseil de la nation Micmac de Gespeg, pour leur soutien financier, matériel, technique et communautaire, ainsi que pour la patience et le temps consacrés à cette recherche.

Nous tenons aussi à remercier le CRSH pour la subvention versée en appui à cette recherche. 